\title{
A-05
}

\section{¿ES POSIBLE MEJORAR LA EFICIENCIA EN EL USO DEL AGUA DE UN VIÑEDO MEDIANTE UN ACOLCHADO ORGÁNICO DEL SUELO?}

\author{
López-Urrea, $R_{.}^{(1)}$, Montoro, $A_{.}^{(2)}$, Martínez, L. ${ }^{(3)}$, Mañas, $F .^{(4)}$, Sánchez, J.M. ${ }^{(5)}$, Intrigliolo,
}

${ }^{1}$ Director, ITAP-FUNDESCAM, Polígono Campollano, Avda. 2ª-42 B, 02007 Albacete. rlu.itap@dipualba.es

2 Investigadora, ITAP, Polígono Campollano, Avda. 2a-42 B, 02007 Albacete. meli.itap@dipualba.es

3 Técnica, FUNDESCAM, Polígono Campollano, Avda. 2a-42 B, 02007 Albacete. Imm.itap@dipualba.es

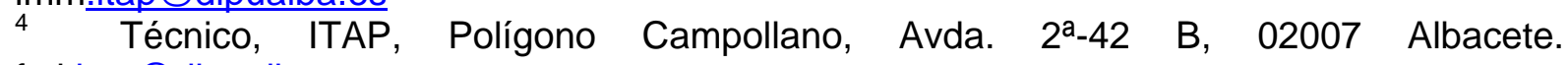
fmj..itap@dipualba.es

5 Profesor, UCLM, Dpto. Física Aplicada, EPC, 16071 Cuenca, juanmanuel.sanchez@uclm.es

${ }^{6}$ Científico Titular, CEBAS-CSIS, Campus Universitario de Espinardo. Espinardo, Murcia. E30100,dintri@cebas.csic.es

\section{Resumen}

El objetivo de este trabajo fue evaluar el efecto que un acolchado orgánico del suelo (restos de poda) puede tener sobre la evapotranspiración del cultivo $\left(E T_{c}\right)$ de la vid con el fin de mejorar la eficiencia en el uso del agua. El estudio se realizó durante la campaña de 2015 en la finca "Las Tiesas", Albacete (España). Las medidas se llevaron a cabo en un lisímetro monolítico de pesada ubicado en el centro de una parcela de vid (Vitis vinífera L., cv. Tempranillo) de 1 ha de superficie, y con cepas plantadas a un marco de 3 x 1,5 m y conducción en espaldera. Se llevaron a cabo tres ciclos de medidas, cada uno consistió en mantener el suelo desnudo durante los dos primeros días, los dos siguientes se cubrió la superficie del lisímetro con un acolchado orgánico (restos de poda de la vid) y los dos últimos días se cubrió el lisímetro con un acolchado inorgánico (lona de plástico). Los resultados indican que para la misma demanda evaporativa y fracción de cubierta vegetal, el acolchado orgánico redujo la $\mathrm{ET}_{\mathrm{c}}$ de la vid algo más de un $17 \%$, mientras que el plástico la redujo un 25\%. Los resultados obtenidos indican que con el acolchado orgánico sigue produciéndose una cierta evaporación de agua desde el suelo, pero se reduce considerablemente la $\mathrm{ET}_{\mathrm{c}}$ pudiéndose mejorar la eficiencia en el uso del agua.

\section{1- Introducción, Objetivos}

El cultivo de la vid ocupa grandes superficies de terreno en la cuenca Mediterránea, así como en otras regiones bajo condiciones climáticas áridas y semiáridas, en las cuales la evapotranspiración de este cultivo es una componente importante del ciclo hidrológico (Trambouze y Voltz, 2001). Castilla-La Mancha es la región que tiene una mayor superficie de viñedo para vinificación en el mundo con 437.072 ha en 2012 (MAGRAMA, 2013), representando algo más del $6 \%$ de la superficie mundial cosechada (FAOSTAT, 2012). Esta región produce 2,75 millones de toneladas de uva y la industria utiliza una parte muy importante de la mano de obra dedicada al sector agrícola en la región. 
En zonas con esta limitación, la prioridad para la agricultura es hacer un uso sostenible del agua, teniendo en cuenta una serie de consideraciones medioambientales, la viabilidad económica, así como ciertos aspectos tecnológicos y sociales (Guerra et al., 2015). Además, la actual escasez de recursos hídricos de muchas zonas de todo el mundo parece puede agravarse en el futuro debido fundamentalmente al cambio climático y la tendencia actual a cambios en la demanda de agua (Skura y Psaltopoulos, 2012).

El manejo del agua en el cultivo de la vid se considera una herramienta importante para mejorar el desarrollo de la planta y la calidad de la uva, así como para conseguir la estabilidad en la producción (Williams y Matthews, 1990). En aquellas zonas donde los recursos hídricos no son un limitante, se puede realizar una programación de riegos basada en producir un cierto estrés durante determinados periodos de tiempo para controlar el desarrollo vegetativo e incrementar la calidad de la uva (Schultz y Matthews, 1993; Jackson y Lombard, 1993). Sin embargo, en zonas áridas y semiáridas donde el agua es un recurso escaso y de gran valor, los viñedos están sometidos frecuentemente a un estrés hídrico muy severo, por lo que se hace necesario un manejo del riego preciso para conseguir una producción sostenible que resulte en una actividad económicamente rentable.

Estudios recientes llevados a cabo en La Mancha Oriental (López-Urrea et al., 2012; Sánchez et al., 2015) indican que la evapotranspiración del cultivo ( $\left.E T_{c}\right)$ de la vid, sin restricción hídrica, medida en un lisímetro de pesada o mediante medidas térmicas y balance de energía se sitúa entre 500 y $550 \mathrm{~mm}$ anuales, siendo la componente evaporativa del balance de agua un $20-30 \%$ del total de la $\mathrm{ET}_{\mathrm{c}}$ de la vid.

El objetivo de este trabajo fue evaluar el efecto que un acolchado orgánico del suelo (restos de poda) puede tener sobre la evapotranspiración del cultivo de la vid con el fin de mejorar la eficiencia en el uso del agua.

\section{2- Materiales y métodos}

El estudio se realizó durante la campaña agrícola de 2015 en la finca "Las Tiesas", Albacete (España). Sus coordenadas geográficas son: $2^{\circ} 5^{\prime}$ ' longitud Oeste, $39^{\circ} 3^{\prime}$ latitud Norte, y $695 \mathrm{~m}$ sobre el nivel del mar. El clima es semiárido, Mediterráneo templado con una precipitación media anual de unos $320 \mathrm{~mm}$, concentrada principalmente en primavera y otoño. Las temperaturas medias, máximas y mínimas son $13,7,24,0$ y $4,5{ }^{\circ} \mathrm{C}$, respectivamente. Para una descripción más detallada del clima de la zona ver López-Urrea et al. (2006).

El suelo de la parcela donde se realizó el estudio se cataloga como Petrocalcic Calcixerepts (Soil Survey Staff, 2006). La profundidad media del suelo de la parcela experimental es de $40 \mathrm{~cm}$ limitado por el desarrollo del horizonte petrocálcico que se encuentra más o menos fragmentado. La textura es franco-arcillo-limosa, con un $13 \%$ de arena, un $49 \%$ de limo y un $38 \%$ de arcilla, con un pH básico. El suelo es pobre en materia orgánica y en nitrógeno, y tiene un alto contenido en caliza activa y potasio.

Las medidas se llevaron a cabo en un lisímetro monolítico de pesada ubicado en el centro de una parcela de viñedo (Vitis vinífera L., cv. Tempranillo) de $100 \times 100 \mathrm{~m}$. La vid fue injertada en 1999 sobre patrón 110 Richter, con un marco de 3 x 1,5 m y conducción en espaldera. Las dimensiones del recipiente del lisímetro son de $3 \times 3 \mathrm{~m}$ de lado y 1,7 $\mathrm{m}$ de profundidad, con un peso total aproximado de 18,5 t. El tanque del lisímetro se apoya sobre un sistema de vigas y un contrapeso que contrarresta la carga muerta del suelo y el tanque del lisímetro, y reduce la carga en la báscula, con un factor de desmultiplicación de 1000:1. Un célula de carga (modelo SB2, Epelsa Ind., S.L., España) está conectada a la báscula. El sistema permite medidas de la evapotranspiración $\left(E T_{c}\right)$ en el lisímetro con una resolución de $250 \mathrm{~g}$, lo que equivale a 0,03 $\mathrm{mm}$ de agua. En el interior del lisímetro se plantaron dos vides, ocupando cada una de ellas $4,5 \mathrm{~m}^{2}$, al igual que el resto de plantas de la parcela de protección, proporcionando una medida representativa de la $E T_{c}$ de la vid.

Las variables meteorológicas fueron medidas durante el experimento con una estación agrometeorológica automática, situada sobre una cubierta de césped y localizada a menos de $100 \mathrm{~m}$ del lisímetro de vid. Los sensores estaban situados entre 1,25 y $2 \mathrm{~m}$ de 
altura por encima de la cubierta de césped y se generaron registros cada quince minutos, horarios y diarios. Las variables medidas fueron: temperatura y humedad relativa del aire (MP100, Campbell Scientific Instrument, Logan, UT, USA), velocidad del viento (A100R, Vector Instruments Ltd., UK), radiaciones de onda corta (CM14, Kipp \& Zonen Delft, Holland), radiaciones de onda larga (CG2, Kipp \& Zonen Delft, Holland) y lluvia (ARG100, Campbell Scientific Instrument, Logan, UT, USA). Toldos los datos registrados se almacenaron en dos dataloggers (CR10X, Campbell Scientific Instrument, Logan, UT, USA). La evapotranspiración de referencia $\left(\mathrm{ET}_{0}\right)$ se calculó utilizando la ecuación de PenmanMonteith FAO56 (P-M FAO56) (Allen et al., 1998). Los valores de coeficiente de cultivo $\left(\mathrm{K}_{\mathrm{c}}\right)$ se obtuvieron dividiendo la $\mathrm{ET}_{\mathrm{C}}$ medida en el lisímetro entre la $\mathrm{ET}_{\mathrm{o}}$ calculada por el método de P-M FAO56.

Se llevaron a cabo tres ciclos de medidas en las siguientes fechas: del 12 al 17 de julio, del 26 al 31 de julio y del 9 al 14 de agosto. Cada ciclo consistió en mantener el suelo desnudo durante los dos primeros días, los dos siguientes se cubrió la superficie del lisímetro con un acolchado orgánico (restos de poda de la vid) y los dos últimos días se cubrió el lisímetro con un acolchado inorgánico (lona de plástico impermeable). Al inicio de cada periodo de 2 días se aplicó un riego de 3 horas (unos $5 \mathrm{~mm}$ ) a las 8:00h am (hora local). De esta forma, se pudo analizar la reducción que se produce en la evaporación (E) con el acolchado orgánico, así como evaluar si con los restos de poda se sigue produciendo algo de evaporación al compararlo con la lona de plástico (evaporación nula). En la Fig. 1 se presentan dos imágenes del lisímetro con los dos tipos de acolchado utilizado. Los valores de $\mathrm{ET}_{\mathrm{c}}$ de la vid se determinaron a partir de las pérdidas de masa en el lisímetro (por evaporación y transpiración) dividido por el área del lisímetro $\left(9 \mathrm{~m}^{2}\right)$. Los registros en que se produjeron ganancias de peso en el lisímetro (por riego), se eliminaron de los cálculos. En total se obtuvieron 462 registros de valores de $\mathrm{ET}_{\mathrm{c}}$ cada quince minutos para cada uno de los ciclos de medida y tipo de suelo (desnudo, restos de poda y plástico).
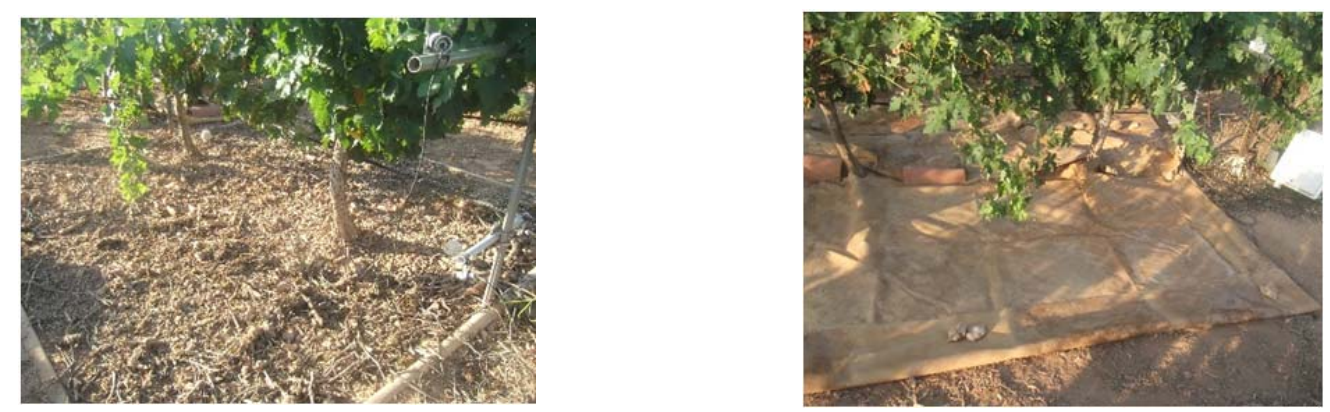

Figura 1. Suelo del lisímetro con restos de poda (izq.) y con una lona de plástico (drcha.).

Durante cada ciclo de medidas se llevaron a cabo una serie de determinaciones en el suelo y las plantas del lisímetro. Se midió de manera continua el contenido de humedad del suelo a 10, 40, 70 y $100 \mathrm{~cm}$ mediante sondas FDR (Frecuence Domain Reflectometry) (EnviroSCANTM, Sentek Pty Ltd., South Australia); los potenciales hídricos de hoja $\left(\Psi_{h}\right)$ y tallo $\left(\Psi_{t}\right)$ se midieron el primer día de cada uno de los tipos de cobertura del suelo (desnudo, restos de poda y plástico) durante los tres ciclos estudiados y a dos horas distintas (11:00 y 13:30 hora local), para lo que se utilizó una cámara de presión tipo Scholander (modelo 600, PMS Instrument Company, Albany, OR, USA); la fracción de suelo cubierta por vegetación $\left(\mathrm{f}_{\mathrm{c}}\right)$ se determinó diariamente a partir de la metodología clásica desarrollada por Cihlar et al. (1987), usando la técnica de la clasificación supervisada de imágenes con el algoritmo de máxima probabilidad, con el fin de asignar las diferentes clases de vegetación verde presentes en la imagen; la conductancia estomática $\left(g_{s}\right)$ se midió con un porómetro (modelo SC-1, Decagon Devices Inc., Pullman, WA, USA) los días indicados para las medidas de potencial hídrico y se llevó a cabo un seguimiento del estado fenológico del cultivo durante cada ciclo de medidas utilizando el código BBCH (Biologische Bundesanstalt, Bundessortenamt and CHemical industry) (Meier, 2001). 


\section{3- Resultados y discusión}

\subsection{Medidas de suelo y planta}

En la Fig. 2 se presenta la evolución del contenido de humedad del suelo a diferentes profundidades durante un ciclo de medidas (12-18 julio). Se observa como la sonda más superficial $(10 \mathrm{~cm})$ responde positivamente a cada uno de los riegos, obteniéndose un contenido de agua en el suelo similar durante todo el periodo estudiado, al igual que en los otros dos ciclos de medidas.

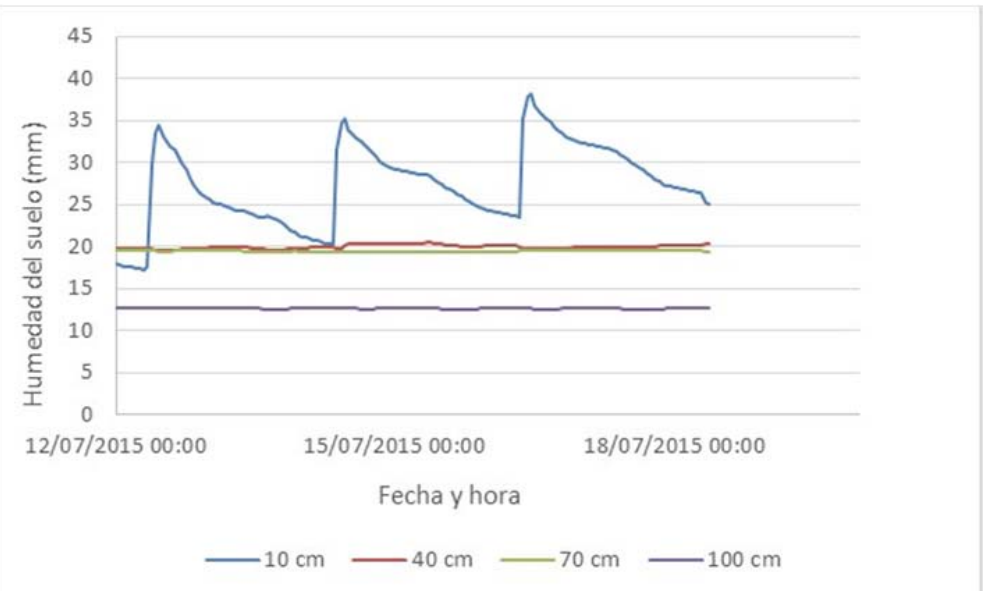

Figura 2. Evolución del contenido de humedad en el suelo a distintas profundidades durante el ciclo del 12 al 18 de julio.

En la Tabla 1 se presenta un resumen de los valores medios de los parámetros medidos en las plantas del lisímetro durante los ciclos estudiados. Los valores de los potenciales hídricos de tallo y hoja a las 13:30h (hora local) indican que las vides en general no padecían de un estrés hídrico acusado en ninguna de las tres condiciones de manejo del suelo. Existe una tendencia a menores valores de potencial hídrico en los días en los que el suelo se cubrió en particular con lona de plástico seguramente debido a la menor $\mathrm{ET}_{\mathrm{c}}$ (resultados mostrados a continuación) y por lo tanto mayor disponibilidad de agua en el suelo. Los resultados de potencial hídrico además sugieren que tanto la lona de plástico como la aplicación de restos de poda posiblemente no variaron notablemente el micro-clima de las cepas dado que los potenciales hídricos no se vieron afectados negativamente por la aplicación de las técnicas de manejo del suelo. La fracción de cubierta vegetal verde fue la misma en los tres ciclos de medidas, indicando que las plantas se encontraban en el mimo estado de desarrollo.

Tabla 1. Valores medios de potenciales de hoja $\left(\Psi_{\mathrm{h}}\right)$ y tallo $\left(\Psi_{\mathrm{t}}\right)$, conductancia estomática $\left(\mathrm{g}_{\mathrm{s}}\right)$, fracción de cubierta vegetal verde $\left(f_{c}\right)$ y fenología del cultivo. 


\begin{tabular}{|c|c|c|c|c|c|c|}
\hline Tipo cubierta & Fecha & $\Psi_{\mathrm{h}}(\mathrm{MPa})$ & $\Psi_{\mathrm{t}}(\mathrm{MPa})$ & $\mathrm{g}_{\mathrm{s}}\left(\mathrm{mmol} \mathrm{m} \mathrm{s}^{-2}\right)$ & $f_{c}$ & Fenología \\
\hline Suelo desnudo & 13-Julio & -1.62 & -1.03 & 343 & 0.32 & Cierre de racimos \\
\hline Restos poda & 15-Julio & -1.55 & -1.03 & 365 & 0.32 & Cierre de racimos \\
\hline Plástico & 17-Julio & -1.50 & -0.93 & 269 & 0.32 & Cierre de racimos \\
\hline Suelo desnudo & 27-Julio & -1.61 & -0.94 & 366 & 0.32 & Envero \\
\hline Restos poda & 29-Julio & -1.64 & -0.96 & 316 & 0.32 & Envero \\
\hline Plástico & 31-Julio & -1.32 & -0.86 & 446 & 0.32 & Envero \\
\hline Suelo desnudo & 10-agosto & -1.66 & -1.00 & 568 & 0.32 & Maduración \\
\hline Restos poda & 12 -agosto & -1.66 & -0.84 & 557 & 0.32 & Maduración \\
\hline Plástico & 14-agosto & -1.56 & -0.80 & 375 & 0.32 & Maduración \\
\hline
\end{tabular}

\subsection{Medidas de evapotranspiración}

En la Fig. 3a se presenta la evolución de la $\mathrm{ET}_{\mathrm{c}}$ de la vid los días 9 de agosto (suelo desnudo), 11 de agosto (restos de poda) y 30 de julio (plástico) después de un riego $(5 \mathrm{~mm})$, teniendo el cultivo un desarrollo vegetativo similar $\left(f_{c}=0,32\right)$ y la misma demanda evaporativa en los distintos días de medida $\left(\mathrm{ET}_{0} \sim 5 \mathrm{~mm}\right.$ día $\left.{ }^{-1}\right)$. En la Fig. 3b se presenta la $\mathrm{ET}_{\mathrm{c}}$ acumulada durante los tres ciclos de medidas para cada uno de los tipos de cubierta de suelo analizados. En ambas figuras se puede observar como los valores de $\mathrm{ET}_{\mathrm{c}}$ son superiores en condiciones de suelo desnudo.
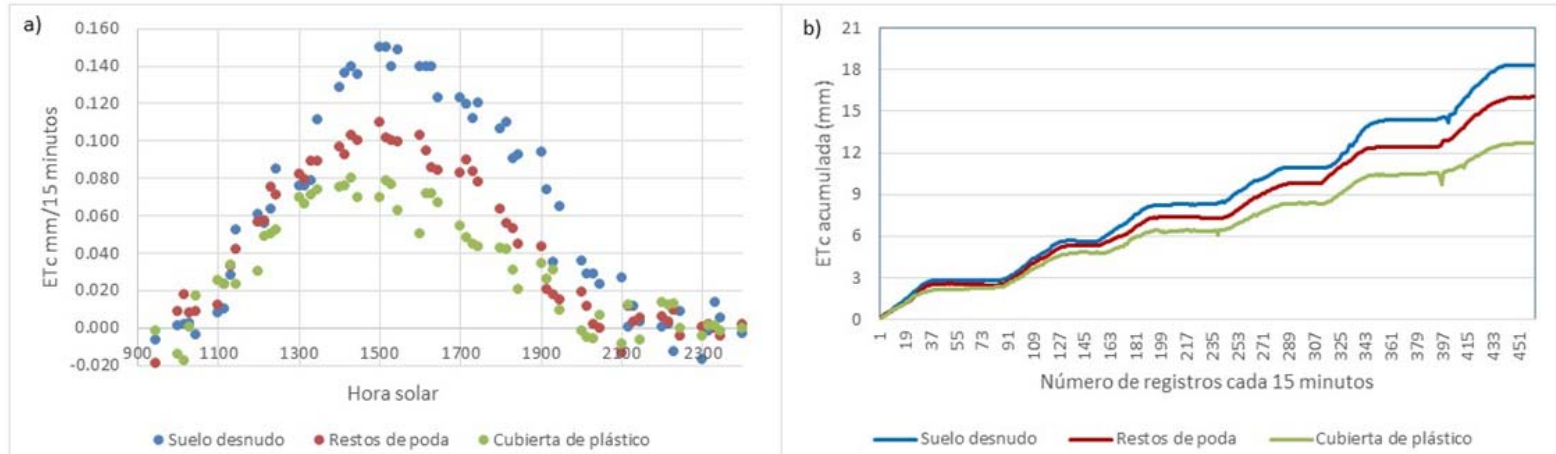

Figura 3. a) Evolución de la evapotranspiración del cultivo $\left(E T_{c}\right)$ cada quince minutos después de un riego; y b) $\mathrm{ET}_{\mathrm{c}}$ acumulada para cada tipo de cubierta de suelo durante el periodo experimental.

En la Tabla 2 se presentan los valores diarios y el total acumulado de $\mathrm{ET}_{\mathrm{c}}$ y $\mathrm{ET}_{\mathrm{o}}$ para los tres tipos de tipo de cubierta. Los resultados indican que para la misma demanda evaporativa y fracción de cubierta vegetal el acolchado orgánico redujo la $E T_{c}$ de la vid algo más de un $17 \%$, mientras que el plástico la redujo un $25 \%$.

Tabla 2. Valores de evapotranspiración de referencia $\left(E T_{0}\right)$ y de cultivo $\left(E T_{c}\right)$, así como sus correspondientes coeficientes de cultivo durante los tres ciclos estudiados. 


\begin{tabular}{lcccc}
\hline Tipo cubierta & Fecha & $\mathrm{ET}_{\mathrm{c}}\left(\mathrm{mm} \mathrm{dí}^{-1}\right)$ & $\mathrm{ET}_{\mathrm{o}}\left(\mathrm{mm} \mathrm{dí}^{-1}\right)$ & Coeficiente de cultivo \\
\hline Suelo & 12-Julio & 2.77 & 5.71 & 0.49 \\
desnudo & 13-Julio & 2.84 & 6.00 & 0.47 \\
& 26-Julio & 2.66 & 5.55 & 0.48 \\
& 27-Julio & 2.62 & 7.52 & 0.35 \\
& 9-Agosto & 3.46 & 4.78 & 0.72 \\
Total & 10-Agosto & 3.93 & 6.95 & 0.57 \\
\hline Restos de & & 18.28 & 36.51 & 0.50 \\
poda & 14-Julio & 2.53 & 6.00 & 0.42 \\
& 15-Julio & 2.81 & 7.09 & 0.40 \\
& 28-Julio & 2.01 & 5.98 & 0.34 \\
& 29-Julio & 2.45 & 7.53 & 0.33 \\
Total & 11-Agosto & 2.61 & 5.05 & 0.52 \\
Plástico & 12-Agosto & 3.62 & 7.05 & 0.51 \\
& & 16.03 & 38.70 & 0.41 \\
\hline & 16-Julio & 2.16 & 4.69 & 0.46 \\
& 17-Julio & 2.67 & 6.76 & 0.39 \\
& 30-Julio & 1.56 & 5.14 & 0.30 \\
& 31-Julio & 1.97 & 5.39 & 0.37 \\
& 13-Agosto & 2.02 & 6.35 & 0.32 \\
& 14-Agosto & 2.31 & 5.38 & 0.43 \\
& & 12.69 & 33.71 & 0.38 \\
\hline \multirow{4}{*}{ Total } & & & &
\end{tabular}

\section{4- Conclusiones y recomendaciones}

En conclusión, los resultados obtenidos indican que a pesar de seguir observándose una cierta evaporación de agua desde el suelo con un acolchado orgánico (alrededor de un $25 \%$ de la $\mathrm{E}$ obtenida con un suelo desnudo), se consigue una reducción de la $E T_{c}$ pudiéndose alcanzar una mejora importante en la eficiencia en el uso del agua (entorno al 20\%).

Se trata de resultados preliminares basados en un año de estudio, por lo que creemos necesario añadir más campañas experimentales al trabajo para obtener resultados más robustos y cuantificar con más solidez el efecto del acolchado sobre la $\mathrm{ET}_{\mathrm{c}}$ del viñedo.

\section{5.- Agradecimientos}

Los autores agradecen la financiación obtenida del Ministerio de Economía y Competitividad a través de los proyectos con co-financiación FEDER AGL2014-54201-C4-4R, RTA 2011-00100-C05-04 y RTA 2014-00049-C05-03

\section{6.- Bibliografía}

Allen, R.G., Pereira, L.S., Raes, D. Smith, M., 1998. Crop evapotranspiration. Guidelines for computing crop water requirements. FAO Irrigation and Drainage, paper $n^{\circ}$. 56, FAO, Rome.

Cihlar, J., Dobson, M.C., Schmugge, T., Hoogeboom, P., Janse, A.R.P., Baret, F., Guyot, G., Le Toan, T., Pampaloni, P., 1987. Procedures for the description of agricultural crops and soils in optical and microwave remote sensing studies. Int. J. Remote Sens. 8, 427-439.

FAOSTAT, 2012. FAO Statistical Database (on line). Consulta: 1 de abril de 2016. http://faostat.fao.org/site/567/DesktopDefault.aspx?PagelD=567\#ancor

Guerra, E., Ventura, F., Snyder, R.L., 2015. Crop Coefficients: A Literature Review. J. Irrig. Drain. Eng., 10.1061/(ASCE)IR.1943-4774.0000983, 06015006. 
Jackson, D., Lombard, P., 1993. Environmental and management practices affecting grape composition and wine quality. A review. Am. J. Enol. Vitic. 44, 409-430.

López-Urrea, R., Martín de Santa Olalla, F., Fabeiro, C. and Moratalla, A. 2006. Testing evapotranspiration equations using lysimeter observations in a semiarid climate. Agric. Water Manage. 85:15-26.

López-Urrea, R., Montoro, A., Mañas, F., López-Fuster, P., Fereres, E., 2012. Evapotranspiration and crop coefficients from lysimeter measurements of mature "Tempranillo" wine grapes. Agricultural Water Management 112, 13-20.

MAGRAMA, 2013. Anuario de Estadística Agraria (on line). Consulta: 1 de abril de 2016. http://www.magrama.gob.es/estadistica/pags/anuario/2013/AE 2013131103 01.p df

Meier, 2001. Growth stages of mono-and dicotyledonous plants. BBCH Monograph. Federal Biological Research Centre for Agriculture and Forestry, $158 \mathrm{pp}$.

Sánchez, J.M., López-Urrea, R., Cuxart, J., Montoro, A., Caselles, V., De la Cruz, F., Doña, C., Martínez, L., 2015. Thermal Remote Sensing Measurements as a Tool to Monitor Evaporation/Transpiration in Vineyards. VIII INTERNATIONAL SYMPOSIUM ON IRRIGATION OF HORTICULTURAL CROPS, Lleida (Spain).

Skuras, D., Psaltopoulos, D., 2012. A broad overview of the main problems derived from climate change that will affect agricultural production in the Mediterranean area." FAO/OECD Workshop: Building Resilience for Adaptation to Climate Change in the Agriculture Sector, FAO, Rome.

Schultz, H., Matthews, M., 1993. Growth, osmotic adjustment, and cell-wall mechanics of expanding grape leaves during water deficits. Crop Sci. 33, 287-294.

Soil Survey Staff, 2006. Keys to Soil Taxonomy, 10th ed. USDA-Natural Resources Conservation Service, Washington, DC.

Williams, L.E., Matthews, M.A., 1990. Grapevine. In: Stewart BA, Nielsen DR (eds) Irrigation of agricultural crops agronomy monograph no. 30. ASA-CSSA-SSSA, Madison, pp 1019-1055 